\title{
CHARACTERIZATION OF MARKOV-BERNOULLI GEOMETRIC DISTRIBUTION RELATED TO RANDOM SUMS
}

\author{
${ }^{1}$ Mohamed Gharib, ${ }^{2}$ Mahmoud M. Ramadan and ${ }^{1}$ Khaled A. H. Al-Ajmi \\ ${ }^{1}$ Department of Mathematics, Faculty of Science, Ain Shams University, Abbasia, Cairo, Egypt \\ ${ }^{2}$ Department of Marketing Administration, Community College, Taif University, Taif, Saudi Arabia \\ ${ }^{2}$ Department of Mathematics, Faculty of Education, Ain Shams University, Roxy, Cairo, Egypt
}

Received 2014-02-12; Revised 2014-03-05; Accepted 2014-03-31

\begin{abstract}
The Markov-Bernoulli geometric distribution is obtained when a generalization, as a Markov process, of the independent Bernoulli sequence of random variables is introduced by considering the success probability changes with respect to the Markov chain. The resulting model is called the MarkovBernoulli model and it has a wide variety of application fields. In this study, some characterizations are given concerning the Markov-Bernoulli geometric distribution as the distribution of the summation index of independent randomly truncated non-negative integer valued random variables. The achieved results generalize the corresponding characterizations concerning the usual geometric distribution.
\end{abstract}

Keywords: Markov-Bernoulli Geometric Distribution, Random Sum, Random Truncation, Characterization

\section{INTRODUCTION}

The Markov-Bernoulli Geometric (MBG) distribution has been obtained by Anis and Gharib (1982) in a study of Markov-Bernoulli sequence of random variables (rv's) introduced by Edwards (1960), who generalized the (usual) independent Bernoulli sequence of rv's by considering the success probability changes with respect to a Markov chain. The resulting model is called the Markov-Bernoulli Model (MBM) or the Markov modulated Bernoulli process (Ozekici, 1997). Many researchers have been studied the MBM from the various aspects of probability, statistics and their applications, in particular the classical problems related to the usual Bernoulli model (Anis and Gharib, 1982; Gharib and Yehia, 1987; Inal, 1987; Yehia and Gharib, 1993; Ozekici, 1997; Ozekici and Soyer, 2003; Arvidsson and Francke, 2007; Omey et al., 2008; Maillart et al., 2008; Pacheco et al., 2009; Cekanavicius and Vellaisamy, 2010; Minkova and Omey, 2011). Further, due to the fact that the MBM operates in a random environment depicted by a Markov chain so that the probability of success at each trial depends on the state of the environment, this model represents an interesting Corresponding Author: Mahmoud M. Ramadan, Department of Marketing Administration, Community College, Taif University Saudi Arabia application of stochastic processes and thus used by numerous authors in, stochastic modeling (Switzer, 1967; 1969; Pedler, 1980; Satheesh et al., 2002; Özekici and Soyer, 2003; Xekalaki and Panaretos, 2004; Arvidsson and Francke, 2007; Nan et al., 2008; Pacheco et al., 2009; Doubleday and Esunge, 2011; Pires and Diniz, 2012).

Let $X_{1}, X_{2}, \ldots$ be a sequence of Markov-Bernoulli rv's with the following matrix of transition probabilities Equation (1.1):

$$
\begin{gathered}
X_{i+1} \\
0 \\
X_{i}{ }_{1}^{0}\left[\begin{array}{cl}
1-(1-\rho) p & (1-\rho) p \\
(1-\rho)(1-p) & \rho+(1-\rho) p
\end{array}\right],
\end{gathered}
$$

and initial distribution:

$$
P\left(X_{1}=1\right)=p=1-P\left(X_{1}=0\right)
$$

where, $0 \leq p \leq 1$ and $0 \leq \rho \leq 1$. 
The sequence $\left\{X_{\mathrm{i}}\right\}$ with the transition matrix (1.1) and the above initial distribution is called the MBM. If $E_{\mathrm{i}}, \mathrm{i}=$ 0,1 are the states of the Markov system given by (1.1), then the parameter $\rho$ which is usually called the persistence indicator of $E_{0}$, is the correlation coefficient between $X_{\mathrm{i}}$ and $X_{\mathrm{i}+1}, i=1,2, \ldots$ (Anis and Gharib, 1982).

If $N$ is the number of transitions for the system defined by (1.1) to be in $E_{1}$ for the first time then $N$ has the MBG distribution given by Equation (1.2):

$$
P(N=k)=p_{k}= \begin{cases}1-\alpha, & k=0 \\ \alpha(1-t) t^{k-1}, & k \geq 1,\end{cases}
$$

where:

$\alpha=1-p$ and $t=\rho+(1-\rho) \alpha=\alpha+(1-\alpha) \rho$.

The MBG distribution (1.2) will be denoted by $\operatorname{MBG}(\alpha, \rho)$ and we shall write $N \sim \operatorname{MBG}(\alpha, \rho)$. Let $P_{\mathrm{U}}(s)=$ $E\left(s^{U}\right)$ be the probability generating function (pgf) of an integer valued random variable $U,|s| \leq 1$.

The pgf of $N$ is given, using (1.2), by Equation (1.3):

$$
P_{N}(s)=\sum_{k=0}^{\infty} p_{k} s^{k}=\frac{(1-\alpha)(1-\rho s)}{(1-t s)} .
$$

Few authors have treated the characterization problem of the MBG distribution (Yehia and Gharib, 1993; Minkova and Omey, 2011).

In this study some new characterizations are given for the MBG distribution by considering it as the distribution of the summation index of a random sum of randomly truncated non-negative integer valued rv's. The scheme of geometric random sum of randomly truncated rv's is important in reliability theory, especially in the problem of optimal total processing time with checkpoints (Dimitrov et al., 1991). The achieved results generalize those given in (Khalil et al., 1991).

\section{RANDOM SUM OF TRUNCATED RANDOM VARIABLES}

Consider a sequence $\left\{X_{n}, n \geq 1\right\}$ of independent identically distributed (iid) non-negative integer valued rv's with probability mass function (pmf) $0 \leq p_{\mathrm{k}}=P\left(X_{1}=\right.$ $k) ; k=0,1,2, \ldots$.

Let $\left\{Y_{\mathrm{n}}, n \geq 1\right\}$ be another sequence of iid nonnegative integer valued random variables, independent of $\left\{X_{\mathrm{n}}\right\}$, with pmf $q_{\mathrm{k}}=P\left(Y_{1}=k\right) ; k=0,1,2, \ldots, \sum_{k=0} q_{k}=1$.
$\left\{Y_{\mathrm{n}}\right\}$ is called the truncating process.

Put $p=P\left(X_{1} \geq Y\right)$ and assume $0<p<1$. Let

$N=\inf \left\{k \geq 1: X_{\mathrm{k}}<Y_{\mathrm{k}}\right\}$. Clearly,
$P(N-1=k)=p^{\mathrm{k}}(1-p), k=0,1,2, \ldots$, i.e., $N-1$ has a geometric distribution with parameter $p$.

\section{Remark 1}

If the sequence $\left\{X_{n}, n \geq 1\right\}$ follows the MBM (1.1), where the event $\left\{\left(X_{\mathrm{i}} \geq Y_{\mathrm{i}}\right)\right\}$ indicates the state $E_{0}$ and the event $\left\{\left(X_{\mathrm{i}}<Y_{\mathrm{i}}\right)\right\}$ indicates the state $E_{1}$, with the initial distribution:

$$
P\left(X_{1} \geq Y_{1}\right)=p \text { and } P\left(X_{1}<Y_{1}\right)=1-p
$$

Then the rv $\mathrm{N}$ (the smallest number of transitions for the system to be in state $E_{1}$ for the first time) wouldhave the MBG distribution defined by (1.2) (Anis and Gharib, 1982) Equation (2.1):

Define:

$Z=\sum_{k=0}^{N-1} Y_{k}+X_{N}$

where, for convenience $Y_{0}=0$. The rv $Z$ is the random sum of truncated rv's (Khalil et al., 1991).

It is known that the $\operatorname{pgf} P_{\mathrm{Z}}(s)$ of $Z$ is given by (Khalil et al., 1991) Equation (2.2 to 2.5):

$$
P_{Z}(s)=P_{1}(s) /\left[1-Q_{1}(s)\right] \text {, }
$$

where:

$$
\begin{aligned}
& P_{1}(s)=E\left[S^{X_{1}} I\left(X_{1}<Y_{1}\right)\right]=\sum_{k=0}^{\infty} p_{k} s^{k} \sum_{\gamma=k+1}^{\infty} q_{\gamma}, \\
& Q_{1}(s)=E\left[S^{Y_{1}} I\left(X_{1} \geq Y_{1}\right)\right]=\sum_{k=0}^{\infty} q_{k} s^{k} \sum_{\gamma=k}^{\infty} p_{\gamma},
\end{aligned}
$$

and $I(A)$ is the indicator function of the set $A$.

\section{Corollary 1}

$$
E(Z)=\frac{\left(\sum_{k=0}^{\infty} k p_{k} \sum_{\gamma=k+1}^{\infty} q_{\gamma}\right)}{\left(1-\sum_{k=0}^{\infty} k q_{k} \sum_{\gamma=k+1}^{\infty} p_{\gamma}\right)}
$$

The result is immediate since $E(\mathrm{Z})=\mathrm{P}^{\prime} \mathrm{Z}(1)$.

\section{Corollary 2}

If the truncating process $\left\{Y_{\mathrm{n}}\right\}$ is such that $Y_{\mathrm{n}} \sim \operatorname{MBG}(\alpha$, $\rho)$, for $\alpha \in(0,1)$ and $\rho \in[0,1]$. Then the $\operatorname{pgf}$ of $Z$ is given by Equation (2.6 and 2.7): 


$$
\begin{aligned}
P_{Z}(s) & =\frac{(1-t s) P_{X_{1}}(t s)}{\left[1-\left\{1-(1-t) P_{X_{1}}(t s)\right\} s\right]} \\
& =\frac{\alpha P_{X_{1}}(t s)}{\left[1-\frac{(1-\alpha)}{(1-t s)}\left\{1-s\left(1-\frac{(1-t)}{(1-\alpha)}\left[1-\alpha P_{X_{1}}(t s)\right]\right)\right\}\right]},
\end{aligned}
$$

and:

$E(Z)=P_{Z}^{\prime}(1)=\frac{\left[1-P_{X_{1}}(t)\right]}{\left[(1-t) P_{X_{1}}(t)\right]}$,

where, $t=\rho+(1-\rho) \alpha$.

\section{Proof}

Since $Y_{\mathrm{n}} \sim \operatorname{MBG}(\alpha, \rho)$, then:

$$
P\left(Y_{n}=k\right)=q_{k}=\left\{\begin{array}{cl}
1-\alpha, & k=0 \\
\alpha(1-t) t^{k-1}, & k=1,2, \ldots .
\end{array}\right.
$$

Hence, using (2.3), we have Equation 2.8 and 2.9:

$$
\begin{aligned}
P_{1}(s) & =\sum_{k=0}^{\infty} p_{k} s^{k} \sum_{\gamma=k+1}^{\infty} q_{\gamma} \\
& =p_{0} \sum_{\gamma=1}^{\infty} q_{\gamma}+\sum_{k=1}^{\infty} p_{k} s^{k} \sum_{\gamma=k+1}^{\infty} q_{\gamma}=\alpha P_{X_{1}}(t s),
\end{aligned}
$$

and, using (2.4), we have:

$$
\begin{aligned}
Q_{1}(s) & =q_{0} \sum_{\gamma=0}^{\infty} p_{\gamma}+\sum_{k=1}^{\infty} q_{k} s^{k} \sum_{\gamma=k}^{\infty} p_{\gamma} \\
& =(1-\alpha)+\alpha(1-t) s \sum_{\gamma=1}^{\infty} p_{\gamma} \sum_{k=1}^{\gamma}(t s)^{k-1} \\
& =\frac{(1-\alpha)}{(1-t s)}\left[1-s\left\{\rho+\alpha(1-\rho) P_{X_{1}}(t s)\right\}\right] .
\end{aligned}
$$

Substituting the expressions of $P_{1}(s)$ and $Q_{1}(s)$ given respectively by (2.8) and (2.9) into (2.2), we get (2.6).

$E(Z)$ is obtained readily from (2.5) or (2.6).

\section{CHARACTERIZATIONS OF THE MBG DISTRIBUTION}

Consider the random sum given by (2.1).

\section{Theorem 1}

Let $Y_{1}$, have a MBG distribution with some parameters $\alpha \in(0,1)$ and $\rho \in[0,1]$ and let $X_{1}$ satisfy $P\left(X_{1}\right.$

Science Publications
$=0)<1$. Then $Z$ has the same distribution as $X_{1}\left(Z \stackrel{d}{=} X_{1}\right)$ if and only if $X_{1}$ has a MBG distribution.

\section{Proof}

Assume that $X_{1} \sim \operatorname{MBG}\left(\beta, \rho_{1}\right)$, for some $\beta \in(0,1)$ and $\rho_{1} \in[0,1]$,i.e.,:

$$
p_{k}= \begin{cases}1-\beta, & k=0 \\ \beta(1-\ell) \ell^{k-1}, & k=1,2, \ldots,\end{cases}
$$

where, $\ell=\rho_{1}+\left(1-\rho_{1}\right) \beta$. Then the $\mathrm{pgf}$ of $X_{1}$ is given by Equation (3.1 and 3.2):

$$
P_{X_{1}}(s)=\frac{(1-\beta)\left(1-\rho_{1} s\right)}{(1-\ell s)} .
$$

Substituting, (3.1) into (2.6), we obtain:

$$
\begin{aligned}
P_{Z}(s) & =\frac{(1-\beta)(1-t s)\left(1-\rho_{1} t s\right)}{1-s\left[1+\ell t-\ell t s-(1-t)(1-\beta)\left(1-\rho_{1} t s\right)\right]} \\
& =\frac{(1-\beta)(1-t s)\left(1-\rho_{1} t s\right)}{t s^{2}\left[\beta+\rho_{1} t(1-\beta)\right]-s\left[\beta+\rho_{1} t(1-\beta)+t\right]+1} \\
& =\frac{(1-\beta)\left(1-\rho_{2} s\right)}{(1-D s)},
\end{aligned}
$$

where, $\rho_{2}=\rho_{1} \mathrm{t}$ and $D=\beta+\rho_{2}(1-\beta)$.

It follows from (3.2), that the rv $Z$ has a MBG distribution with parameters $\beta \in(0,1)$ and $\rho_{2} \in[0,1]$.

Conversely, assume that $P_{\mathrm{Z}}(\mathrm{s})=P_{X_{1}}(s)$ where $Y_{1} \sim \operatorname{MBG}(\alpha, \rho)$. Hence, we have to show that $P_{X_{1}}(s)$ is the pgf of MBG distribution. Replacing $P_{\mathrm{Z}}(s)$ by $P_{X_{1}}(s)$ in (2.6), yields in: $(1-t s) P_{X_{1}}\left(t s\left[1-s\left\{1-(1-t) P_{X_{1}}(t s)\right\}\right]=\right.$ $P_{X_{1}}(s)$. Or: $(1-t s) P_{X_{1}}(t s)=(1-s) P_{X_{1}}(s)+s(1-t) P_{X_{1}}(s) P_{X-}$ ${ }_{1}(t s)$.

Dividing both sides of this equation by $(1-s)(1-t s)$ $P_{X_{1}}(s) P_{X_{1}}(t s)$, we get:

$$
\frac{1}{(1-t s) P_{X_{1}}(t s)}+\frac{s(1-t)}{(1-s)(1-t s)}=\frac{1}{(1-s) P_{X_{1}}(s)} .
$$

Now, using partial fractions for the middle term and using some manipulations, we can write Equation (3.3):

$$
\frac{\mathrm{P}_{\mathrm{X}_{1}}^{-1}(\mathrm{ts})-1}{(1-\mathrm{ts})}=\frac{\mathrm{P}_{\mathrm{X}_{1}}^{-1}(\mathrm{~s})-1}{(1-\mathrm{s})}
$$

JMSS 
Or, $H(t s)=H(s)$, where, $H(s)=\frac{P_{X_{1}}^{-1}(s)-1}{(1-s)}$.

Putting $s=1$, we get:

$$
H(t)=H(1)=\lim _{s \rightarrow 1} \frac{P_{X_{1}}^{-1}(s)-1}{(1-s)}=E\left(X_{1}\right)=C, \text { say }
$$

Therefore, the solution of (3.3) is given by:

$$
P_{X_{1}}(s)=[1+C(1-s)]^{-1} \text {. }
$$

Choosing $C=\beta /(1-\beta)\left\{1-[\alpha+(1-\alpha) \rho] \rho_{1}\right\}$ and recalling that $\alpha+(1-\alpha) \rho=t$, we finally get:

$$
P_{X_{1}}(s)=\frac{(1-\beta)\left(1-\rho_{1} s\right)}{(1-\ell s)}
$$

where, $\ell=\rho_{1}+\left(1-\rho_{1}\right) \beta$.

Which is the pgf of the $\operatorname{MBG}\left(\beta, \rho_{1}\right)$ distribution. Hence $X_{1} \sim \operatorname{MBG}\left(\beta, \rho_{1}\right)$.

This completes the proof of Theorem 1 .

\section{Remark 2}

It follows from Theorem 1 that when $X_{1}$ has the MBG distribution then the random sum $Z$ and the summands have distributions of the same type and in this case the summands are called $N$-sum stable (Satheesh et al., 2002). This result is valid, also, as a consequence of the fact that geometric random sums are stable in the same sense.

Another characterization for the MBG distribution can be obtained in terms of the expected values of $\mathrm{Z}$ and $X_{1}$.

\section{Theorem 2}

Let $Y_{1}$ have a MBG distribution with parameters $\alpha \in(0,1)$ and $\rho \in[0,1]$ and consider $A=\{(\alpha, \rho): \alpha \in(0,1)$, $\rho \in[0,1]\}$. Then $X_{1}$ has a $\operatorname{MBG}\left(\beta, \rho_{1}\right)$ distribution if and only if $\left.\forall(\alpha, \rho) \in A, E(Z)=\beta /(1-\beta)\left(1-\rho_{2}\right)\right]$, where $\rho_{2}=\rho_{1} t$ and $t=\rho+(1-\rho) \alpha$.

\section{Proof}

If $X_{1} \sim \operatorname{MBG}\left(\beta, \rho_{1}\right)$ for some $\left(\beta, \rho_{1}\right) \in A$, then it follows from Theorem 1 that $Z \sim \operatorname{MBG}\left(\hat{a}, \rho_{2}\right)$, where $\rho_{2}=\rho_{1} t$ and $t=\rho+(1-\rho) \alpha$. Consequently, $E(Z)=\beta /\left[(1-\beta)\left(1-\rho_{2}\right)\right]$.
Conversely, suppose that: $E(Z)=\beta /\left[(1-\beta)\left(1-\rho_{2}\right)\right]$ for $\operatorname{some}\left(\beta, \rho_{1}\right) \in A$.

Then from (2.7), we have:

$$
\frac{\beta}{(1-\beta)\left(1-\rho_{2}\right)}=\frac{1-P_{X_{1}}(t)}{(1-t) P_{X_{1}}(t)} \text {. }
$$

Solving this equation with respect to $P_{X_{1}}(s)$, one has:

$$
P_{X_{1}}(t)=\frac{(1-\beta)\left(1-\rho_{1} t\right)}{(1-\ell t)}, \forall(\alpha, \beta) \in A .
$$

Which is the pgf of the MBG $\left(\beta, \rho_{1}\right)$ distribution. Hence $X_{1} \sim \operatorname{MBG}\left(\beta, \rho_{1}\right)$.

This completes the proof of Theorem 2 .

The following theorem expresses the relation between the distribution of $\mathrm{Z}$ and the distribution of the truncating process $\left\{Y_{\mathrm{n}}\right\}$.

\section{Theorem 3}

Let $X_{1}$ have a $\operatorname{MBG}\left(\beta, \rho_{1}\right)$ distribution for some parameters $\beta \in(0,1)$ and $\rho_{1} \in[0,1]$ and let $Y_{1}$ satisfy $q_{0}$ $=P\left(Y_{1}=0\right)<1$. Then $Z^{d}=X_{1}$ if and only if $Y_{1}$ follows a MBG distribution.

\section{Proof}

Since $X_{1} \sim \operatorname{MBG}\left(\beta, \rho_{1}\right)$, for some $\beta \in(0,1)$ and $\rho \in[0,1]$, then:

$$
p_{k}= \begin{cases}1-\beta, & k=0 \\ \beta(1-\ell) \ell^{k-1} & k=1,2, \ldots\end{cases}
$$

where, $\ell=\rho_{1}+\left(1-\rho_{1}\right) \beta$.

Proceeding as in Corollary 2, one has:

$$
\begin{aligned}
P_{1}(s) & =\sum_{k=0}^{\infty} p_{k} s^{k} \sum_{\gamma=k+1}^{\infty} q_{\gamma} \\
& =(1-\beta)\left(1-q_{0}\right)+\beta(1-\ell) s \sum_{\gamma=2}^{\infty} q_{\gamma} \sum_{k=1}^{\gamma-1}(\ell s)^{k-1} \\
& =\frac{(1-\beta)}{\ell(1-\ell s)}\left[\ell-\rho_{1} q_{0}-\ell s \rho_{1}\left(1-q_{0}\right)-\beta\left(1-\rho_{1}\right) P_{Y_{1}}(\ell s)\right],
\end{aligned}
$$

and: 


$$
\begin{aligned}
\mathrm{Q}_{1}(\mathrm{~s}) & =\sum_{\mathrm{k}=0}^{\infty} \mathrm{p}_{\mathrm{k}} \mathrm{s}^{\mathrm{k}} \sum_{\gamma=\mathrm{k}}^{\infty} \mathrm{p}_{\gamma} \\
& =\mathrm{q}_{0}+\frac{\beta}{\ell} \sum_{\mathrm{k}=1}^{\infty} \mathrm{q}_{\mathrm{k}}(\ell \mathrm{s})^{\mathrm{k}}=\frac{1}{\ell}\left[\beta \mathrm{P}_{\mathrm{Y}_{1}}(\ell \mathrm{s})+\mathrm{q}_{0} \rho_{\mathrm{Y}_{1}}(1-\beta)\right]
\end{aligned}
$$

Substituting (3.4) and (3.5) into (2.2), we obtain:

$$
\begin{aligned}
P_{Z}(s) & =\frac{(1-\beta)}{(1-\ell s)}\left[\frac{\ell-\rho_{1} q_{0}-\ell \rho_{1}\left(1-q_{0}\right) s-\beta\left(1-\rho_{1}\right) P_{Y_{1}}(\ell s)}{\ell-\rho_{1} q_{0}(1-\beta)-\beta P_{Y_{1}}(\ell s)}\right] \\
& =\frac{(1-\beta)}{(1-\ell s)}\left[\frac{\rho_{1}+\left(1-\rho_{1}\right) \beta-\rho_{1} q_{0}-\ell \rho_{1}\left(1-q_{0}\right) s-\beta\left(1-\rho_{1}\right) P_{Y_{1}}(\ell s)}{\rho_{1}(1-\beta)\left(1-q_{0}\right)+\beta\left(1-P_{Y_{1}}(\ell s)\right)}\right]
\end{aligned}
$$

Now assume that $Z \stackrel{d}{=} X_{1}$, then $P_{Z}(s)=P_{X_{1}}(\mathrm{~s})$.

Consequently, equating (3.1) and (3.6) and then solving for $P_{Y_{1}}(\ell s)$, we get:

$$
P_{Y_{1}}(\ell s)=\frac{\beta q_{0}+\left[\rho_{1}\left(1-q_{0}\right)-\beta\right] s}{\beta(1-s)}
$$

From which we finally have:

$$
P_{Y_{1}}(s)=\frac{q_{0}\left\{1-\left(\rho q_{0}\right)^{-1}\left[1-\rho_{1} \beta^{-1}\left(1-q_{0}\right)\right] s\right\}}{\left(1-\ell^{-1} s\right)} .
$$

Which is the pgf of a $\operatorname{MBG}\left(\gamma, \rho_{2}\right)$ distribution with,

$$
\gamma=1-q_{0} \text { and } \rho_{2}=\left(\rho q_{0}\right)\left[1-\rho_{1} \beta^{-1}\left(1-q_{0}\right)\right] \text {. }
$$

Hence $Y_{1} \sim \operatorname{MBG}\left(\gamma, \rho_{2}\right)$.

The "only if" part of the proof follows directly by applying Theorem 1 .

This completes the proof of Theorem 3.

\section{Remark 3}

The results of (Khalil et al., 1991) follow as special cases from our corresponding results when the MBM (1.1) reduces to the independence case by putting the correlation parameter $\rho=0$.

\section{CONCLUSION}

In this study three characterizations for the MarkovBernoulli geometric distribution are proved. These results extend the corresponding characterizations of the geometric distribution. Further, the achieved results have a direct relevance to the stability problem of random sums of random variables. Moreover, the given characterization theorems will be useful, in

understanding the missing link between the mathematical structure of Markov-Bernoulli geometric distribution and the actual behavior of some real world random phenomena.

\section{REFERENCES}

Anis, A.A. and M. Gharib, 1982. On the Bernoulli Markov sequences. Proceedings of the 17th Annual Conference Stat. Math. Inst. Stat. Studies Res. Dec 13-16, Cairo University Press, Cairo, Egypt, pp: 1-21.

Arvidsson, H. and S. Francke, 2007. Dependence in nonlife insurance. U.U.D.M. Project Report, 23. Department of Mathematics.

Cekanavicius, V. and P. Vellaisamy, 2010. Compound Poisson and signed compound Poisson approximations to the Markov binomial law. Bernoulli, 16: 1114-1136.

Dimitrov, B., Z. Khalil, N. Kolev and P. Petrov, 1991. On the optimal total processing time using checkpoints. IEEE Trans. Software Eng., 17: 436442. DOI: $10.1109 / 32.90446$

Doubleday, K. J. and J. N. Esunge, 2011. Application of Markov chains to stock trends. J. Math. Stat., 7: 103-106. DOI: 10.3844/jmssp.2011.103.106

Edwards, A.W.F., 1960. The meaning of binomial distribution. Nature, 186: 1074. DOI: 10.1038/1861074a0

Gharib, M. and A.Y. Yehia, 1987. A limit theorem for the sum of n-Markov-Bernoulli random variables. Egypt. Stat. J., 31: 53-61.

Inal, C., 1987. On the limiting distribution for Bernoulli trials within a Markov-chain context. Commun. Fac. Sci. Univ. Ank. Ser. A, 36: 123-129.

Khalil, Z., B. Dimitrov and J.P. Dion, 1991. A characterization of the geometric distribution related to random sums. Commun. Stat.-Stochastic Models, 7: 321-326. DOI: 10.1080/15326349108807192

Maillart, L.M., C.R. Cassady and J. Honeycutt, 2008. A binomial approximation of lot yield under Markov modulated Bernoulli item yield. IIE Trans., 40: 459467. DOI: 10.1080/07408170701592507

Minkova, L.D. and E. Omey, 2011. A new Markov binomial distribution. HUB Res. Econ. Manage.

Nan, F., Y. Wang and X. Ma, 2008. Application of multiscale hidden Markov modelling Wavelet coefficients to fMRI activation detection. J. Math. Statist., 4: 255-263. DOI: 10.3844/jmssp.2008.255.263 
Omey, E., J. Santos and S.V. Gulck, 2008. A Markovbinomial distribution. Applicable Anal. Discrete Math., 2: 38-50. DOI: 10.2298/AADM0801038O

Ozekici, S. and R. Soyer, 2003. Bayesian analysis of Markov modulated Bernoulli processes. Math. Methods Operat. Res., 57: 125-140. DOI: $10.1007 / \mathrm{s} 001860200268$

Ozekici, S., 1997. Markov modulated Bernoulli process. Math. Methods Operat. Res., 45: 311-324. DOI: 10.1007/BF01194782

Pacheco, A., L.C. Tang, N.U. Prabhu, 2009. MarkovModulated Processes and Semiregenerative Phenomena. 1st Edn., World Scientific Pub Co Inc., UK. ISBN-10: 9812793186, pp: 224.

Pedler, P.J., 1980. Effect of dependence on the occupation time in a two-state stationary Markov chain. J. Am. Statist. Associat., 75: 739-746. DOI: 10.1080/01621459.1980.10477545

Pires, R.M. and C.A.R. Diniz, 2012. Correlated binomial regression models. Comput. Statist. Data Analysis, 56: 2513-2525. DOI: 10.1016/j.csda.2012.02.004
Satheesh, S., N.U. Nair and E. Sandhya, 2002. Stability of random sums. Stochastic Modelling Applic., 5: 17-26.

Switzer, P., 1967. Reconstructing patterns from sample data. Ann. Math. Statist., 38: 138-154. DOI: 10.1214/aoms/1177699064

Switzer, P., 1969. Mapping a geographically correlated environment. 1st Edn., Defense Technical Information Center, pp: 38.

Xekalaki, E. and J. Panaretos, 2004. A binomial distribution with dependent trials and its use in stochastic model evaluation. Commun. Stat. Theory Meth., 33: 3043-3058.

Yehia, A.Y. and M. Gharib, 1993. Characterizations of the Markov-Bernoulli geometric distribution. Micro. Reliab., 33: 497-499. 